PROCEEDINGS OF THE

AMERICAN MATHEMATICAL SOCIETY

Volume 131, Number 5, Pages 1553-1556

S 0002-9939(02)06715-1

Article electronically published on September 19, 2002

\title{
INTERPOLATION INEQUALITIES IN BESOV SPACES
}

\author{
SHUJI MACHIHARA AND TOHRU OZAWA
}

(Communicated by Andreas Seeger)

Dedicated to Professor Takaaki Nishida on the occasion of his sixtieth birthday

\begin{abstract}
In this paper we present an interpolation inequality in the homogeneous Besov spaces on $\mathbb{R}^{n}$, which reduces to a number of well-known inequalities in special cases.
\end{abstract}

\section{INTRODUCTION}

There are several types of interpolation inequalities in the Sobolev and Besov spaces on $\mathbb{R}^{n}$; see for instance [1]-[15] and references therein. We prove the following theorem (see below for notation).

Theorem 1. Let $\lambda, \mu, p, q, r, \theta$ satisfy $\lambda, \mu \in \mathbb{R}, 1 \leq p, q \leq r \leq \infty, 0<\theta<1$,

$$
\begin{aligned}
& \lambda>\frac{n}{p}-\frac{n}{r}, \\
& \mu<\frac{n}{q}-\frac{n}{r}, \\
& \theta\left(\lambda-\frac{n}{p}+\frac{n}{r}\right)+(1-\theta)\left(\mu-\frac{n}{q}+\frac{n}{r}\right)=0 .
\end{aligned}
$$

Then there exists a constant $C>0$ such that

$$
\left\|f ; \dot{B}_{r, 1}^{0}\right\| \leq C\left\|f ; \dot{B}_{p, \infty}^{\lambda}\right\|^{\theta}\left\|f ; \dot{B}_{q, \infty}^{\mu}\right\|^{1-\theta}
$$

for all $f \in \dot{B}_{p, \infty}^{\lambda} \cap \dot{B}_{q, \infty}^{\mu}$.

By the embeddings $\dot{B}_{r, 1}^{0} \hookrightarrow L^{r}$ and $\dot{H}_{r}^{\rho} \hookrightarrow \dot{B}_{r, \infty}^{\rho}$ with $1 \leq r \leq \infty, \rho \in \mathbb{R}$, we have the following corollary.

Corollary 2. Let $\lambda, \mu, p, q, r, \theta$ be as above. Then there exists a constant $C>0$ such that

$$
\left\|f ; L^{r}\right\| \leq C\left\|f ; \dot{H}_{p}^{\lambda}\right\|^{\theta}\left\|f ; \dot{H}_{q}^{\mu}\right\|^{1-\theta}
$$

for all $f \in \dot{H}_{p}^{\lambda} \cap \dot{H}_{q}^{\mu}$.

Received by the editors July 10, 2001 and, in revised form, December 27, 2001.

2000 Mathematics Subject Classification. Primary 46B70, 46M35.

(C) 2002 American Mathematical Society 
The results above generalize various previously known interpolation inequalities. In [9], Miyakawa proved (1.5) in the special cases: (a) $r=\infty, \lambda=\mu$; (b) $r=$ $\infty, p=q, \mu=0$. In [5], Escobedo and Vega proved (1.5) in the special case: (c) $r=\infty, p, q>1,0<\lambda, \mu<n$. Corollary 2 ensures that (1.5) holds when $\mu \leq 0$. In [11, (1.5) is proved in the special case: (d) $r=\infty, p=q=2$.

There are some available interpolation inequalities not covered by the results above. Complex interpolation yields (1.5) with $1<p, q, r<\infty, 1 / r=$ $(1-\theta) / p+\theta / q,(1-\theta) \lambda+\theta \mu=0$ (see also 7]), and therefore (1.1) and (1.2) amount to additional restrictions. The known complex interpolation formulas for Besov spaces do not cover (1.4), however. A possible dependence of the third index on the interpolation inequalities is a novelty of (1.4) (see also [8, 12]).

We know a few more results which seem to be related to (1.4) and (1.5). In [6], Gérard, Meyer, and Oru proved

$$
\left\|f ; L^{r}\right\| \leq C\left\|f ; \dot{H}_{p}^{\lambda}\right\|^{p / r}\left\|f ; \dot{B}_{\infty, \infty}^{-\alpha}\right\|^{1-p / r},
$$

where $1<p<r<\infty, \alpha(r / p-1)=\lambda>0$, in particular, $p=2, r=6, \lambda=1, \alpha=$ 1/2. In [4], Cohen, Dahmen, Daubechies, and De Vore proved

$$
\left\|f ; L^{2}\right\| \leq C\|f ; B V\|^{1 / 2}\left\|f ; B_{\infty, \infty}^{-1}\right\|^{1 / 2},
$$

where $B V$ denotes the space of functions vanishing at infinity in the weak sense and satisfying the estimate

$$
\sup _{y \in \mathbb{R}^{n}}|y|^{-1} \int|f(x+y)-f(x)| d x \leq C .
$$

We prove the theorem in the next section. The proof depends on the standard technique from the Littlewood-Paley theory (see [1, 2, 3, 6, 8, 11, 15, for instance) and therefore the theorem holds for the homogeneous Besov spaces on the Heisenberg group $\mathbb{H}^{n}$ with necessary modifications (see [1]).

We finally introduce the notation. For any $r$ with $1 \leq r \leq \infty, L^{r}=L^{r}\left(\mathbb{R}^{n}\right)$ denotes the Lebesgue space on $\mathbb{R}^{n}$. For any $\rho \in \mathbb{R}$ and any $r$ with $1 \leq r \leq$ $\infty, \dot{H}_{r}^{\rho}$ denotes the homogeneous Sobolev space defined as the space of classes of distributions $f$ modulo polynomials such that $(-\Delta)^{\rho / 2} f \in L^{r}$, where $\Delta$ is the Laplacian in $\mathbb{R}^{n}$. For any $\rho \in \mathbb{R}^{n}$ and any $r, m$ with $1 \leq r, m \leq \infty, \dot{B}_{r, m}^{\rho}$ denotes the homogeneous Besov space defined as the space of classes of distributions $f$ modulo polynomials such that $\left\{2^{\rho j}\left\|\varphi_{j} * f ; L^{r}\right\|\right\} \in l^{m}(\mathbb{Z})$, where $*$ denotes the convolution in $\mathbb{R}^{n}$ and the Fourier transformed functions $\left\{\hat{\varphi}_{j}\right\} \subset C_{0}^{\infty}$ satisfy $\sum_{j \in \mathbb{Z}} \hat{\varphi}_{j}(\xi)=1$ for all $\xi \in \mathbb{R}^{n} \backslash\{0\}, 0 \leq \hat{\varphi}_{j} \leq 1, \operatorname{supp} \hat{\varphi}_{j} \subset\left\{\xi ; 2^{j-1} \leq|\xi| \leq 2^{j+1}\right\}, \hat{\varphi}_{j}(\xi)=\hat{\varphi}_{0}\left(2^{-j} \xi\right)$. We refer to [2, 3, 7, 15, for general information on homogeneous Besov and TriebelLizorkin spaces.

\section{Proof of the theorem}

We may assume that $\left\|f ; \dot{B}_{p, \infty}^{\lambda}\right\| \neq 0$ and $\left\|f ; \dot{B}_{q, \infty}^{\mu}\right\| \neq 0$. From the support properties of $\hat{\varphi}_{j}$ it follows that

$$
\left\|f ; \dot{B}_{r, 1}^{0}\right\|=\sum_{j \in \mathbb{Z}}\left\|\varphi_{j} * f ; L^{r}\right\| \leq \sum_{j \in \mathbb{Z}} \sum_{k=j-1}^{j+1}\left\|\varphi_{k} * \varphi_{j} * f ; L^{r}\right\| .
$$


By the Young inequality, we have

$$
\begin{aligned}
\left\|\varphi_{k} * \varphi_{j} * f ; L^{r}\right\| & \leq\left\|\varphi_{k} ; L^{m}\right\|\left\|\varphi_{j} * f ; L^{s}\right\| \\
& =2^{n k(1-1 / m)}\left\|\varphi_{0} ; L^{m}\right\|\left\|\varphi_{j} * f ; L^{s}\right\|,
\end{aligned}
$$

where $1+1 / r=1 / m+1 / s$. We apply (2.2) with $s=p, q$ to (2.1) to obtain

$$
\begin{aligned}
\left\|f ; \dot{B}_{r, 1}^{0}\right\| \leq & C \sum_{j \geq l} 2^{(n / p-n / r-\lambda) j} \cdot 2^{\lambda j}\left\|\varphi_{j} * f ; L^{p}\right\| \\
& +C \sum_{j<l} 2^{(n / q-n / r-\mu) j} \cdot 2^{\mu j}\left\|\varphi_{j} * f ; L^{q}\right\| \\
\leq & C \sum_{j \geq l} 2^{(n / p-n / r-\lambda) j}\left\|f ; \dot{B}_{p, \infty}^{\lambda}\right\|+C \sum_{j<l} 2^{(n / q-n / r-\mu) j}\left\|f ; \dot{B}_{q, \infty}^{\mu}\right\| \\
\leq & C\left(2^{(n / p-n / r-\lambda) l}\left\|f ; \dot{B}_{p, \infty}^{\lambda}\right\|+2^{(n / q-n / r-\mu) l}\left\|f ; \dot{B}_{q, \infty}^{\mu}\right\|\right) \\
= & C\left(2^{(n / p-n / r-\lambda) l} a^{1-\theta}+2^{(n / q-n / r-\mu) l} a^{-\theta}\right)\left\|f ; \dot{B}_{p, \infty}^{\lambda}\right\|^{\theta}\left\|f ; \dot{B}_{q, \infty}^{\mu}\right\|^{1-\theta},
\end{aligned}
$$

where $a=\left\|f ; \dot{B}_{p, \infty}^{\lambda}\right\| /\left\|f ; \dot{B}_{q, \infty}^{\mu}\right\|$.

Let $\sigma=(\lambda-n / p+n / r)-(\mu-n / q+n / r)>0$ and let $l$ be the largest integer that is less than or equal to $\sigma^{-1} \log _{2} a$. Then,

$$
2^{l} \leq a^{1 / \sigma} \leq 2 \cdot 2^{l}, \quad \theta=-(\mu-n / q+n / r) / \sigma, \quad 1-\theta=(\lambda-n / p+n / r) / \sigma,
$$

and therefore

$$
\begin{gathered}
2^{(n / p-n / r-\lambda) l} a^{1-\theta} \leq\left(2 a^{-1 / \sigma}\right)^{\lambda-n / p+n / r} a^{1-\theta}=2^{\lambda-n / p+n / r}, \\
2^{(n / q-n / r-\mu) l} a^{-\theta} \leq a^{(n / q-n / r-\mu) / \sigma} a^{-\theta}=1 .
\end{gathered}
$$

This proves the theorem.

\section{ACKNOWLEDGments}

We wish to thank the referee for enlightening suggestions.

\section{REFERENCES}

[1] H. Bahouri, P. GÉrard and C. -J. Xu, Espace de Besov et estimations de Strichartz généralisées sur le group de Heisenberg, J. d'Anal. Math., 82 (2000), 93-118. MR 2001k:58054

[2] J. Bergh And J. Löfström, Interpolation spaces, Springer, Berlin/Heiderberg/New York (1976). MR 58:2349

[3] P. Brenner, V. Thomée and L. Wahlbin, Besov spaces and applications to difference methods for initial value problems, Lecture Notes in Math., 434, Springer-Verlag, Berlin/Heidelberg/New York (1975). MR 57:1106

[4] A. Cohen, W. Dahmen, I. Daubechies and R. De Vore Harmonic analysis of the space $B V$, Preprint, RWTH Aachen, Institut für Geometrie und Praktische Mathematik, Nr. 195 (2000) (see http://www.igpm.rwth-aachen.de).

[5] M. Escobedo and L. Vega, A semilinear Dirac equation in $H^{s}\left(\mathbb{R}^{3}\right)$ for $s>1$, SIAM J. Math. Anal., 28 (1997), 338-362. MR 97k:35239

[6] P. Gérard, Y. Meyer and F. Oru, Inégalités de Sobolev précisées, Seminaire de EDP, Ecole Polytechnique, 1996-1997. CMP 98:04

[7] J. Ginibre And G. Velo, The global Cauchy problem for the nonlinear Klein-Gordon equation, Math. Z., 189 (1985), 487-505. MR 86f:35149

[8] H. Kozono, T. Ogawa And Y. TAniuchi, Critical Sobolev inequalities and application to the semilinear evolution equations (preprint).

[9] T. Miyakawa, On Morrey spaces of measures: Basic properties and potential estimates, Hiroshima Math. J., 20 (1990), 213-222. MR 91k:46021 
[10] Y. MEYER, Oscillating patterns in image processing and in some nonlinear evolution equations, The Fifteenth Dean J.B. Lewis Memorial Lectures (2001).

[11] M. Nakamura and T. OzaWa, Small solutions to nonlinear Schrödinger equations in the Sobolev spaces, J. d'Anal. Math., 81 (2000), 305-329. MR 2001j:35256

[12] T. Ogawa And Y. Taniuchi, Critical Sobolev inequality and its application to nonlinear evolution equations in the fluid mechanics, RIMS Kokyuroku, 1162 (2000), 99-106. CMP 2001:05

[13] T. Ozawa, On critical cases of Sobolev's inequalities, J. Funct. Anal., 127 (1995), 259-269. MR 96c: 46039

[14] T. OzaWA, Characterization of Trudinger's inequality, J. Inequal. \& Appl., 1 (1997), 369374. MR 2001e:46061

[15] H. Triebel, Theory of Function Spaces, Birkhäuser, Basel (1983). MR 86j:46026

Department of Mathematics, Hokkaido University, Sapporo 060-0810, Japan

Current address: Department of Mathematics, Shimane University, Matsue, Shimane 6908504, Japan

E-mail address: machihara@math.shimane-u.ac.jp

Department of Mathematics, Hokkaido University, Sapporo 060-0810, Japan 